\title{
Erratum to: Immunomodulation after ischemic stroke: potential mechanisms and implications for therapy
}

Cynthia Santos Samary ${ }^{1}$, Paolo Pelosi ${ }^{2}$, Pedro Leme Silva ${ }^{1}$ and Patricia Rieken Macedo Rocco ${ }^{{ }^{*}}$

Following publication of this article [1] it came to our attention that we failed to reference a review article by Shim and Wong [2] which influenced our review for Critical Care. We sincerely apologize for this oversight.

\footnotetext{
Author details

${ }^{1}$ Laboratory of Pulmonary Investigation, Carlos Chagas Filho Biophysics, Institute, Federal University of Rio de Janeiro, Centro de Ciências da Saúde, Avenida Carlos Chagas Filho, s/n, Bloco G-014, Itha do Fundão, 21941-902 Rio de Janeiro, RJ, Brazil. '2Department of Surgical Sciences and Integrated Diagnostics, IRCCS AOU San Martino-IST, University of Genoa, Genoa, Italy.
}

Received: 4 September 2017 Accepted: 4 September 2017

Published online: 18 October 2017

\section{References}

1. Samary CS, Pelosi P, Silva PL, Rocco PRM. Immunomodulation after ischemic stroke: potential mechanisms and implications for therapy. Crit Care. 2016:20:391.

2. Shim R, Wong CHY. Ischemia, Immunosuppression and Infection-Tackling the Predicaments of Post-Stroke Complications. Int J Mol Sci. 2016;17:64. 